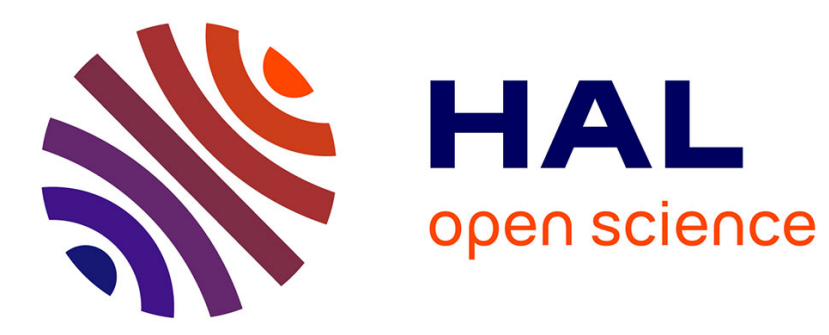

\title{
Frequency selection in globally unstable round jets
}

Lutz Lesshafft, Patrick Huerre, Pierre Sagaut

\section{To cite this version:}

Lutz Lesshafft, Patrick Huerre, Pierre Sagaut. Frequency selection in globally unstable round jets. Physics of Fluids, 2007, 19 (5), pp.054108. 10.1063/1.2732247 . hal-01023085

\section{HAL Id: hal-01023085 \\ https://hal-polytechnique.archives-ouvertes.fr/hal-01023085}

Submitted on $20 \mathrm{Jul} 2014$

HAL is a multi-disciplinary open access archive for the deposit and dissemination of scientific research documents, whether they are published or not. The documents may come from teaching and research institutions in France or abroad, or from public or private research centers.
L'archive ouverte pluridisciplinaire HAL, est destinée au dépôt et à la diffusion de documents scientifiques de niveau recherche, publiés ou non, émanant des établissements d'enseignement et de recherche français ou étrangers, des laboratoires publics ou privés. 


\title{
Frequency selection in globally unstable round jets
}

\author{
Lutz Lesshafft ${ }^{\text {a) }}$ and Patrick Huerre \\ Laboratoire d'Hydrodynamique (LadHyX), CNRS-École Polytechnique, 91128 Palaiseau, France
}

Pierre Sagaut

d'Alembert Institute, Université Pierre et Marie Curie-Paris 6, 4 Place Jussieu, 75252 Paris Cedex 05, France

(Received 13 December 2006; accepted 23 March 2007; published online 21 May 2007)

\begin{abstract}
The self-sustained formation of synchronized ring vortices in hot subsonic jets is investigated by direct numerical simulation of the axisymmetric equations of motion. The onset of global instability and the global frequency of synchronized oscillations are examined as functions of the ambient-to-jet temperature ratio and the initial jet shear layer thickness. The numerical results are found to follow the predictions from nonlinear global instability theory; global instability sets in as the unperturbed flow is absolutely unstable over a region of finite streamwise extent at the inlet, and the global frequency near the global instability threshold corresponds to the absolute frequency of the inlet profile. In strongly supercritical thin shear layer jets, however, the simulations display global frequencies well above the absolute frequency, in agreement with experimental results. The inner structure of rolled-up vortices in hot jets displays fine layers of positive and negative vorticity that are produced and maintained by the action of the baroclinic torque. (C) 2007 American Institute
\end{abstract} of Physics. [DOI: 10.1063/1.2732247]

\section{INTRODUCTION}

Axisymmetric jets have been experimentally observed to sustain self-excited large-scale vortices, synchronized at a well-defined frequency, if the jet is sufficiently heated with respect to the ambient air (Monkewitz et al. ${ }^{1}$ ). Such selfexcited oscillations are the manifestation of a global instability of the unperturbed steady flow. Subjected to an arbitrary perturbation, a globally unstable steady flow will bifurcate and settle into a new organized regime of highly regular oscillations. This new state is termed a global mode of the underlying steady flow, and its oscillations are tuned to a well-defined global frequency. In a large variety of open shear flows, the occurrence of global instability has been shown to be closely connected to the local linear instability properties (see Huerre and Monkewitz ${ }^{2}$ and $\mathrm{Chomaz}^{3}$ for reviews). If the unperturbed flow is locally convectively unstable everywhere, externally induced perturbations are amplified, but the flow is globally stable. In the absence of continuous forcing, it will ultimately return to a steady state. In the presence of a locally absolutely unstable flow region, in contrast, the flow may bifurcate to a global mode. Prominent examples of flows exhibiting global instability triggered by local absolute instability include the cylinder wake, ${ }^{4-7}$ counterflowing shear layers, ${ }^{8}$ swirling jets, ${ }^{9}$ and jets with counterflow. ${ }^{10,11}$

In the hot jet experiments of Monkewitz et al., ${ }^{1}$ selfsustained synchronized oscillations were found to set in as the ambient-to-jet temperature ratio $S=T_{\infty} / T_{c}$ was lowered below a critical value of 0.73 . The Strouhal number of these oscillations is given as $\mathrm{St} \sim 0.3$, based on jet diameter and

\footnotetext{
${ }^{a)}$ Present address: Department of Mechanical Engineering, University of California, Santa Barbara, CA 93106; Electronic mail: Lutz.Lesshafft@ladhyx.polytechnique.fr
}

exit velocity. At temperature ratios below 0.63 , a second oscillating state with $\mathrm{St} \sim 0.45$ was observed to be dominant. Both of these modes were axisymmetric. Kyle and Sreenivasan $^{12}$ (see also Ref. 13) investigated the stability of mixed helium/air jets in ambient air. The jet-to-ambient density ratio that served as a control parameter in these experiments is equivalent, under the perfect gas assumption, to the ambient-to-jet temperature ratio used in Ref. 1. The experiments of Kyle and Sreenivasan ${ }^{12}$ showed only one oscillating state, also axisymmetric, with Strouhal number and density ratio ranges in good agreement with the $\mathrm{St} \sim 0.45$ mode of Monkewitz et al. ${ }^{1}$ More recent helium jet experiments ${ }^{14,15}$ confirm the results of Kyle and Sreenivasan. ${ }^{12}$

The linear spatio-temporal instability analysis of Monkewitz and Sohn ${ }^{16}$ revealed that hot axisymmetric jets display a region of absolute instability as the temperature ratio falls below a critical value. In the inviscid, zero-Mach-number limit, this critical value is $S=0.72$, in striking agreement with the first threshold value $S=0.73$ at which global instability was observed experimentally. ${ }^{1}$

Further theoretical studies by Jendoubi and Strykowski ${ }^{11}$ demonstrated that two distinct axisymmetric spatio-temporal instability modes exist in round jets: a shear layer mode, similar to the Kelvin-Helmholtz instability mode in a plane shear layer, and a jet column mode, identical to the one discovered by Monkewitz and Shon, ${ }^{16}$ with maximum pressure perturbations on the jet axis. In jets without counterflow, the absolute instability mode is of the jet column type. A recent linear analysis (Lesshafft and Huerre ${ }^{17}$ ) has revealed that the transition from convective to absolute instability in variabledensity jets, even in the absence of gravity, ensues from the action of the baroclinic torque.

Theoretical discussions of the experimental results on global instability in variable-density jets have so far essen- 
tially been restricted to a comparison of the temperature or density ratio threshold values for the observed onset of selfsustained oscillations with the predicted onset of absolute instability in inviscid, zero-Mach-number parallel flow. For the particular value $S=0.48$, Kyle and Sreenivasan ${ }^{12}$ also compared the measured global frequencies with theoretical values of the absolute frequency given in Ref. 16. Rather good agreement was found as long as the jet shear layer at the nozzle exit was not too thin. For very thin shear layers however, as well as for lower values of $S$, the comparison was much less favorable. Furthermore, the shear layer thickness was determined from a measured boundary layer velocity profile at the nozzle exit in the experiments, ${ }^{12}$ and from an analytical free jet profile in the theoretical study of Monkewitz and Sohn. ${ }^{16}$

Theoretical analyses of the frequency selection process in globally unstable semi-infinite flows, such as jets, have been carried out by Couairon and Chomaz ${ }^{18,19}$ on the basis of Ginzburg-Landau model equations. Their criterion for a semi-infinite parallel base flow states that nonlinear global instability coincides with the onset of linear absolute instability, and that the selected global frequency at this threshold is given by the absolute frequency of the base flow. In supercritical flows, the absolute frequency only provides a leading order prediction of the global frequency. However, the numerical simulations by Chomaz ${ }^{20}$ have demonstrated that, in parallel wakes, this zero-order criterion yields highly accurate predictions far into the supercritical regime. The theoretical analysis has been extended to account for slow streamwise variations of the base flow. ${ }^{5}$ In such cases, the global frequency at the global instability threshold is still given by the absolute frequency at the upstream boundary. However, global instability is expected to set in only if the region of absolute instability is sufficiently large to allow for a nonlinear saturation of the oscillation amplitude.

The objective of the present study is to numerically explore the applicability of nonlinear global mode theory $5,18,19$ to the self-sustained synchronized oscillations in hot jets. A recent investigation ${ }^{21}$ has established that the theoretical predictions of Couairon and Chomaz 5 accurately match numerical simulation results for a family of heated thick shear layer jets. The present study extends the investigated parameter range to approach the experimental settings of Monkewitz et $a .^{1}{ }^{2}$ and Sreenivasan et al. ${ }^{13}$

The paper is organized as follows: In Sec. II, the physical flow model is presented, and the different flow parameters are specified. Numerical aspects such as the computational grid, boundary and initial conditions are discussed in Sec. III. Further details on the employed flow solver can be found in Ref. 21. Numerical results are presented in Sec. IV. A comparison with experiments ${ }^{1}$ is included, and the role of acoustic feedback is discussed. Section V describes observations on the inner structure of rolled-up vortex billows in strongly heated jets. The main results are summarized in Sec. VI.

\section{PROBLEM FORMULATION}

Consider an axisymmetric subsonic jet of radius $R$ issuing into a quiescent ambient medium. The flow is assumed to be governed by the axisymmetric compressible NavierStokes equations and the equation of state for a perfect gas, cast in cylindrical coordinates $x$ and $r$. These equations are given explicitly in Lesshafft $e t a l^{21}$ The system is formulated in terms of conservative flow variables $\boldsymbol{q}=(\rho, \rho u, \rho v, \rho E)$, where $\rho$ is density, $u$ and $v$ are the axial and radial velocity components, respectively, and $E$ is the total energy.

The total quantities $\boldsymbol{q}$ are separated into a base flow $\boldsymbol{q}_{b}(x, r)$ and a perturbation component $\boldsymbol{q}^{\prime}(x, r, t)$. By construction, any given base flow $\boldsymbol{q}_{b}$ is a steady state solution of the governing equations. As in Ref. 21, the streamwise development of the base flow is obtained as a numerical solution of the compressible boundary layer equations, starting at $x=0$ from the analytical velocity profile ${ }^{22}$

$$
u_{b}(r)=\frac{1}{2}+\frac{1}{2} \tanh \left[\frac{R}{4 \theta}\left(\frac{1}{r}-r\right)\right]
$$

of momentum shear layer thickness $\theta$. All quantities are made nondimensional with respect to the jet radius $R$, velocity $U_{c}$, density $\rho_{c}$, and temperature $T_{c}$, where the subscript $c$ denotes the value on the jet centerline in the inlet plane. The dynamic viscosity $\mu$ and the thermal conductivity $\kappa$ are taken as constant throughout the flow, and gravity is neglected. A specific flow configuration is then defined by the following set of parameters: the Reynolds number $\mathrm{Re}$ $=\rho_{c} R U_{c} / \mu$, the Mach number Ma $=U_{c} / c_{c}$ with respect to the speed of sound $c_{c}$ on the jet centerline, the Prandtl number $\operatorname{Pr}=\mu c_{p} / \kappa$, with $c_{p}$ the specific heat at constant pressure, the ambient-to-jet temperature ratio $S=T_{\infty} / T_{c}$, the inlet velocity profile parameter $R / \theta$, and the ratio of specific heats $\gamma$ $=c_{p} / c_{v}$.

The flow parameters considered in the numerical study are chosen to closely correspond to settings of Monkewitz et al. ${ }^{1}$ The Reynolds number is taken as $\mathrm{Re}=3750\left(\mathrm{Re}_{D}\right.$ $=7500$, based on jet diameter as in Ref. 1) and the Mach number as $\mathrm{Ma}=0.1$. Values of $\operatorname{Pr}=1$ and $\gamma=1.4$ are retained throughout. The occurrence of global instability is then explored for combinations of the inlet profile parameter and the temperature ratio over the ranges $10 \leqslant R / \theta \leqslant 25$ and 0.3 $\leqslant S \leqslant 1$.

\section{NUMERICAL METHOD}

The numerical procedure used in the simulations is identical to that of Lesshafft et al. ${ }^{21}$ An algorithm described by $\mathrm{Lu}$ and Lele ${ }^{23}$ is used to obtain the base flow $\boldsymbol{q}_{b}$ by numerical integration of the boundary layer equations. The temporal evolution of perturbations $\boldsymbol{q}^{\prime}$ within this base flow is then computed directly from the axisymmetric Navier-Stokes equations. Spatial derivatives are evaluated from a sixthorder accurate explicit centered finite-difference scheme, and 
the solution is advanced in time via a third-order RungeKutta algorithm. Numerical stability of the finite-difference formulation is achieved by applying a tenth-order explicit filter scheme at each time step. ${ }^{24,25}$

The orthogonal grid that has been used in all computations discretizes the physical domain $0 \leqslant x \leqslant 30$ and $0<r$ $\leqslant 30$ into $500 \times 278$ grid points. In the radial direction, these points are concentrated in the shear layer region around $r$ $=1$, with a minimum spacing $\Delta r_{\min }=0.01$. In the axial direction, $\Delta x$ is kept at 0.05 for $x \leqslant 15$, and then is slowly increased up to $\Delta x=0.1$ at $x=25$. Sponge regions are introduced at the lateral and downstream boundaries of the physical domain, where grid stretching is gradually increased up to a rate of $4 \%$. These sponge regions extend over 30 $\leqslant r \leqslant 112$ and $30 \leqslant x \leqslant 125$, discretized with 37 radial and 200 axial grid points. A damping term $-\lambda(x, r) \boldsymbol{q}^{\prime}$ is added to the Navier-Stokes equations within the sponge regions. The value of the damping coefficient $\lambda(x, r)$ is smoothly ramped up from zero at the boundary of the physical domain to 0.3 at $x=50$ and $r=50$, according to a function given by Chomaz. ${ }^{20}$ Convergence tests on finer grids $(750 \times 410$ points $)$ and on larger computational domains have confirmed that the results are grid independent and unaffected by box effects.

The theoretical model for global modes in a semi-infinite domain studied by Couairon and Chomaz, ${ }^{5}$ to which our numerical simulation results are to be compared, assumes a nonlinear wave front blocked at a Dirichlet-type upstream boundary that lies within an absolutely unstable flow region. Such a configuration precludes the implementation of an additional sponge region at the upstream boundary of the computational domain, which then would be penetrated by the upstream-traveling wave front up to the streamwise station where artificial damping induces a transition to convective instability. Instead, as in Ref. 21, the characteristic boundary conditions of Giles ${ }^{26}$ are used at the inlet. These are designed to provide a first order correction for oblique incidence of upstream-traveling acoustic waves. Tests have shown that this correction significantly reduces the coupling of acoustic and vortical disturbances at the upstream boundary. Numerical instabilities due to these boundary conditions have been reported by Colonius et al. ${ }^{27}$ but they have not been encountered in the present simulations.

A different set of characteristic upstream boundary conditions, without any correction for oblique incidence of acoustic waves, was employed in an earlier study. ${ }^{28}$ The global dynamics observed in the simulations are quite sensitive to the quality of the upstream boundary conditions. It must be pointed out that the use of the term "first order" in the context of the boundary conditions given by Giles ${ }^{26}$ is ambiguous in the literature. In compliance with Ref. 27, we will henceforth denote as "first order" boundary conditions those used in Ref. 21 as well as in the present study. The boundary conditions used in Ref. 28, which assume acoustic wave fronts to be parallel to the upstream boundary, will be denoted as being of "zero order."

All computations start from a divergence-free initial velocity perturbation in the form of a vortex ring ${ }^{29}$

$$
\begin{aligned}
& u^{\prime}(r, x)=-A \frac{r_{0}\left(r-r_{0}\right)}{r s} g(r, x), \\
& v^{\prime}(r, x)=A \frac{r_{0}\left(x-x_{0}\right)}{r s} g(r, x), \\
& g(r, x)=\exp \left[-\ln 2 \frac{\left(x-x_{0}\right)^{2}+\left(r-r_{0}\right)^{2}}{s^{2}}\right],
\end{aligned}
$$

with $\left(r_{0}, x_{0}\right)=(1,2), s=0.3$ and $A=0.1$. We have found that the jet column mode, which alone may give rise to the growth of a nonlinear global mode induced by absolute instability, is most efficiently excited by a high-amplitude initial pulse that quickly leads to vortex roll-up. That way, a strong exponential temporal growth of shear layer modes is bypassed, and the jet-column mode grows to nonlinear saturation within a short time interval.

In Sec. IV, the numerical results are compared to predictions drawn from a linear stability analysis of the underlying base flow. A detailed description of the numerical method used to solve the linear instability problem is given in Ref. 17.

\section{ONSET AND FREQUENCY OF SELF-SUSTAINED OSCILLATIONS}

\section{A. Numerical observations and comparison with theoretical predictions}

The long-time flow behavior, after the transient wave packet induced by the initial perturbation (2) has left the computational domain, is radically distinct for globally stable and globally unstable situations. For subcritical combinations of $R / \theta$ and $S$, only low-level broadband fluctuations persist near the inlet, followed by irregular vortex roll-up and pairing events. At sufficiently high values of $R / \theta$ and strong heating, the flow settles into a highly periodic oscillatory state. The jet shear layer in these cases rolls up at a fixed streamwise station, forming a street of regularly spaced ring vortices that are slowly attenuated as they travel downstream. Periodic states obtained in two typical configurations are visualized in Fig. 1. Note that the rolled-up vortices in the thin shear layer jet [Fig. 1(b)] undergo one pairing event around $x=13$. In the present simulations, precisely one such stage of vortex pairing is observed in all globally unstable cases with inlet profiles $R / \theta>10$. Vortex pairing in the asymptotic flow state shows the same high degree of repeatability as the initial vortex roll-up.

The qualitative difference between synchronized and nonsynchronized asymptotic states is demonstrated in the spatio-temporal diagrams in Fig. 2. The thinnest shear layer jet considered in this study, with temperature ratios just above and below the critical value, is chosen as an example. The radial perturbation velocity $v^{\prime}(r=1, x, t)$ at the center of the shear layer is presented as a function of streamwise distance and time. In order to clearly visualize all flow regions, $v^{\prime}$ has been normalized independently at each streamwise station $x$ with respect to its maximum amplitude over the displayed time interval in Fig. 2. 


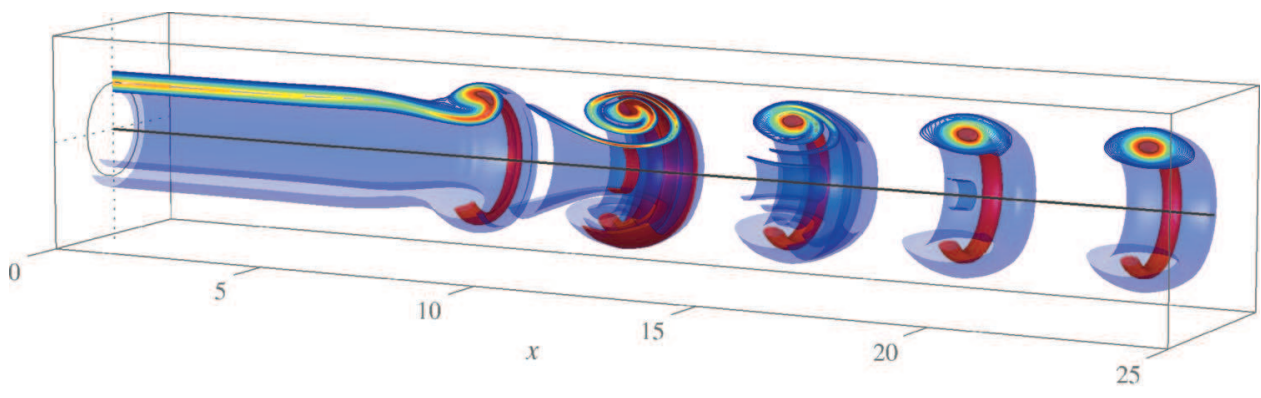

a) $R / \theta=10, S=0.4$

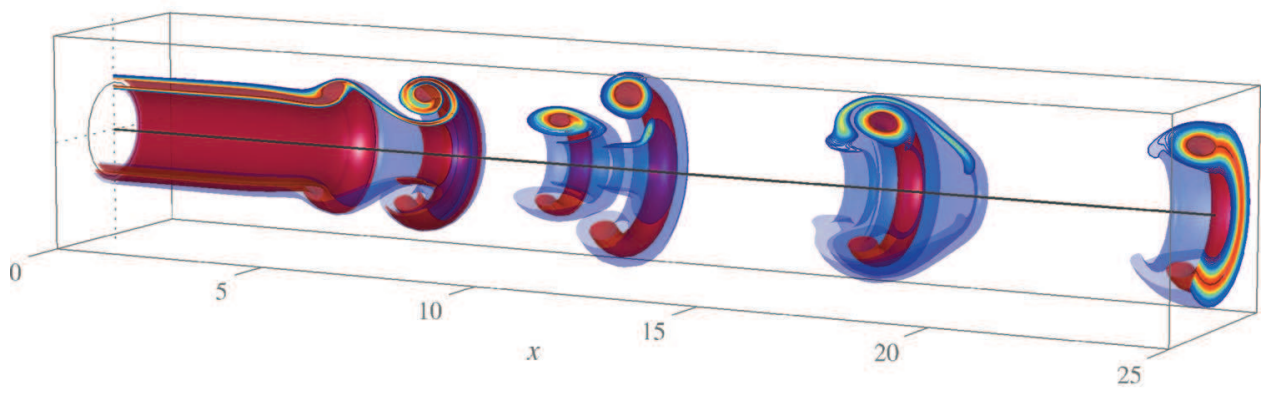

FIG. 1. Snapshots of synchronized oscillations in two globally unstable jets. Vorticity isosurfaces $\Omega=1$ (blue) and $\Omega=3$ (red), isocontours $1 \leqslant \Omega \leqslant 3$. (a) Thick shear layer jet, no vortex pairing; (b) thin shear layer jet, with vortex pairing.
The corresponding Strouhal number spectra, computed from $v^{\prime}(r=1, x=1, t)$, are displayed in Fig. 3. As in Monkewitz et al. ${ }^{1}$ the Strouhal number is defined as $\mathrm{St}=2 f R / U_{c}$. In the globally stable configuration with $S=0.65$ (thin line), the spectrum is broadband. As the base flow in this case is con-

(a)

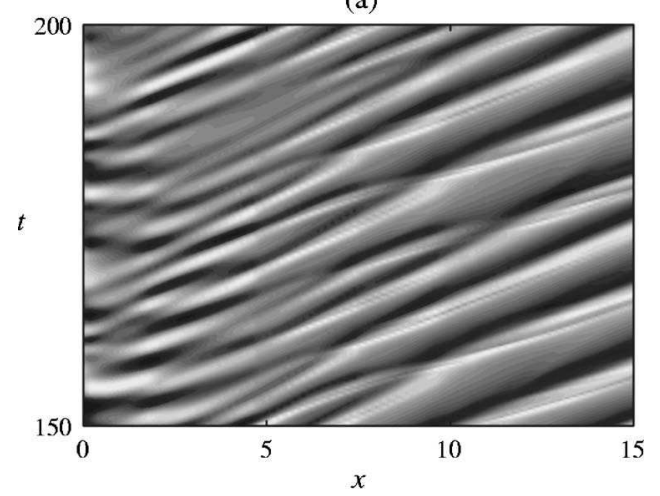

(b)

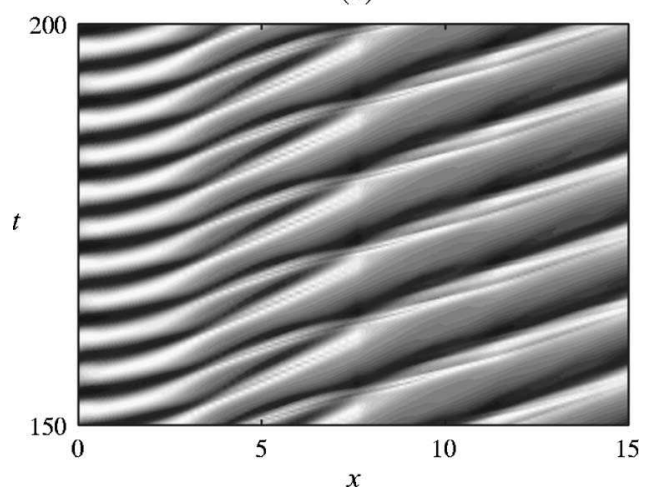

FIG. 2. Spatio-temporal diagrams of the radial perturbation velocity $v^{\prime}(r=1, x, t)$ in the long-time regime. (a) Globally stable case $R / \theta=25$, $S=0.65$; (b) globally unstable case $R / \theta=25, S=0.55$. vectively unstable throughout the entire physical domain, persisting perturbations at long times can only arise from a continuous, spurious excitation at the numerical inlet boundary. This excitation is caused by upstream-travelling acoustic waves that in turn are emitted from the downstream vortices. The jet shear layer then acts as a bandpass filter, promoting the growth of the most amplified spatial instability modes. In contrast, the spectrum of the synchronized flow case (thick line in Fig. 3) is marked by sharp peaks, while the background noise level is significantly lowered. As the spectra are taken near the upstream boundary, the dominant peak corresponds to the fundamental global frequency $\mathrm{St}_{g}$ of vortex roll-up. A subharmonic peak in the spectrum of the synchronized case announces the occurrence of vortex pairing further downstream. Some peaks in the line-dominated spectrum in Fig. 3, e.g., at $\mathrm{St}=1.1$, are accompanied by small "side peaks" on both sides. Whether these are the result of a sideband instability, ${ }^{30}$ or just an effect of the FFT algorithm

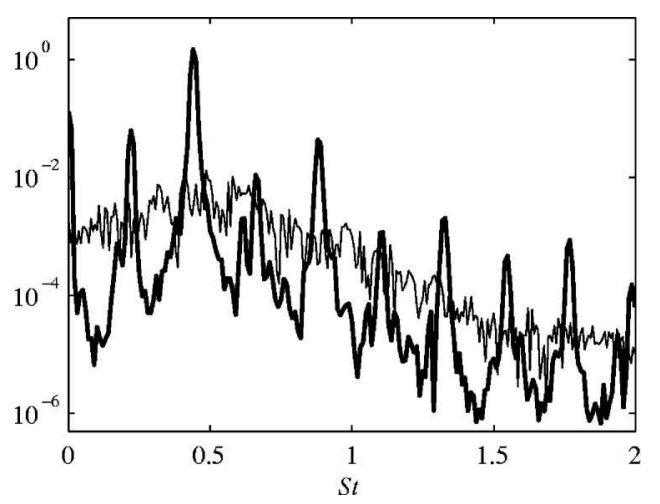

FIG. 3. Spectral density of $v^{\prime}(r=1, x=1, t)$ as a function of Strouhal number for the two configurations of Fig. 2. Thin line: globally stable case $R / \theta$ $=25, S=0.65$; thick line: globally unstable case $R / \theta=25, S=0.55$. 


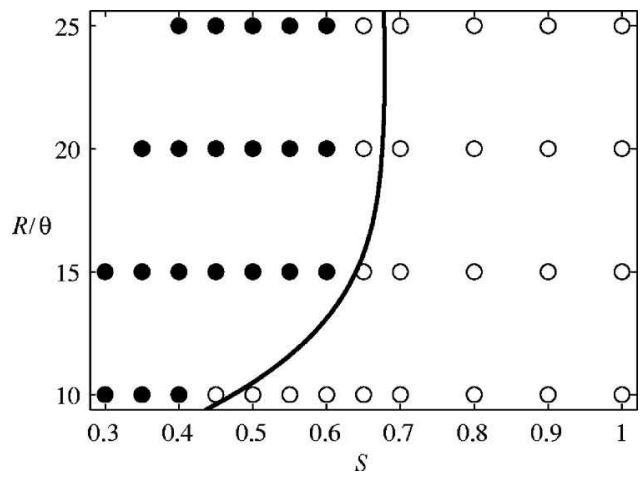

FIG. 4. State diagram of hot jets: synchronized $(\bullet)$ and nonsynchronized $(\bigcirc)$ asymptotic states observed over the investigated ranges of temperature ratio $S$ and inlet profile parameter $R / \theta$; absolute/convective instability boundary of inlet profile $(-)$.

(a Hanning windowing technique has been used), cannot be decided at present.

The globally unstable region of the $S-R / \theta$ parameter plane is identified in Fig. 4. Flow cases exhibiting selfsustained synchronized oscillations are marked as solid circles, whereas open circles represent nonsynchronized cases. The black line marks the absolute/convective instability boundary of the inlet profile, computed for $\mathrm{Ma}=0.1$ and $\operatorname{Re}=3750$. Base flows in the parameter region to the left of this boundary display absolute instability at the inlet, and they do so over an increasingly large streamwise interval for stronger heating and thinner initial shear layers. Figure 4 clearly demonstrates that global instability is detected only in base flows with an absolutely unstable inlet profile. The boundary of global instability in the $S-R / \theta$ plane closely follows that of local absolute instability, with a slight offset into the absolutely unstable parameter region. In full agreement with the theoretical predictions of Couairon and Chomaz $^{5}$ as well as with earlier numerical simulations, ${ }^{21}$ the present results confirm that global instability occurs only in the presence of a sufficiently large pocket of local absolute instability.

Due to the high quality of the flow synchronization, the global frequency can be determined by measuring the oscillation period $\tau$ directly from the temporal signal $v^{\prime}(r=1$, $x=1, t)$ of the asymptotic state. The temporal development of $\mathrm{St}=2 / \tau$ is shown in Fig. 5 for the three globally unstable cases encountered at $S=0.5$. The asymptotic values $\mathrm{St}_{g}$ in these cases are converged to at least three significant digits. Corresponding FFT frequency spectra of the $v^{\prime}$ signal, similar to the thick line in Fig. 3, are fully consistent with the results obtained for $\mathrm{St}_{g}$ from Fig. 5, but they would require much larger signal samples of the asymptotic regime in order to give the same accuracy.

The model analysis of Couairon and Chomaz ${ }^{5}$ predicts the frequency of a global mode in a semi-infinite domain to correspond to the absolute frequency at the upstream boundary in the limit of marginal global instability. As the base flow becomes increasingly supercritical, the global frequency may depart from this leading-order criterion. In Fig. 6, values of the global frequency $\mathrm{St}_{g}$, observed in the numerical simulations, are compared to the Strouhal number $\mathrm{St}_{0}$ of the

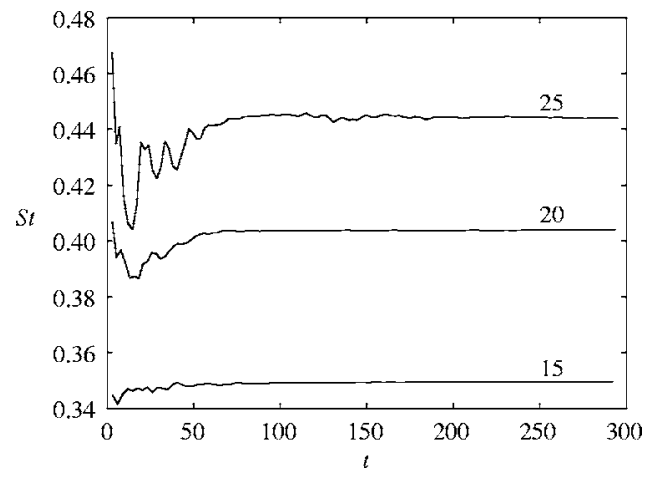

FIG. 5. Temporal development of the oscillation Strouhal number in jets at $S=0.5$. Labels indicate the value of $R / \theta$.

absolute instability mode, obtained from a linear stability analysis of the inlet base flow profile. For each $R / \theta$, values of $\mathrm{St}_{0}$ are given over the absolutely unstable range of temperature ratios. Experimental measurements from Monkewitz et al. ${ }^{1}$ are also shown and will be discussed in Sec. IV C.

The thickest shear layer jet with $R / \theta=10$ synchronizes to the absolute frequency within $0.5 \%$ at its global instability threshold $S=0.4$. At supercritical temperature ratios, $\mathrm{St}_{g}$ gradually shifts to values slightly higher than $\mathrm{St}_{0}$. The same

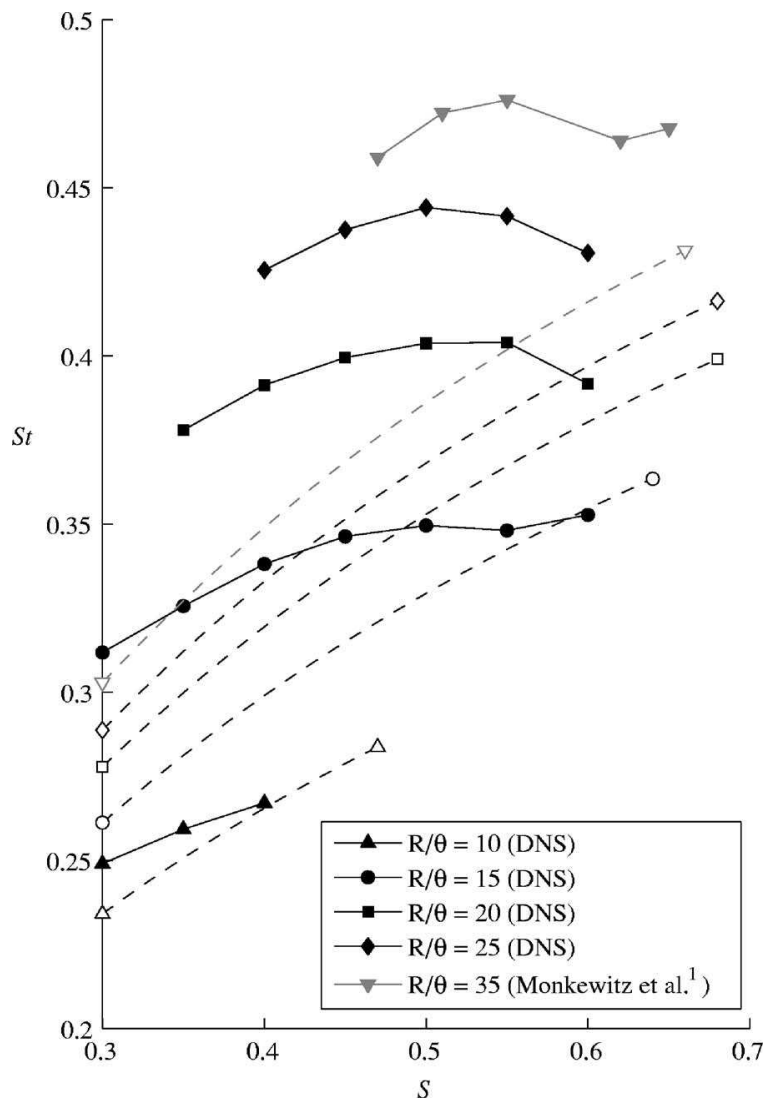

FIG. 6. Global frequency compared to absolute frequency of the inlet profile, both as functions of the temperature ratio $S$ for various values of the inlet profile parameter $R / \theta$. Black solid lines: global frequencies observed in the present simulations; grey solid line: experimental values from Monkewitz et al. (Ref. 1); dashed lines and open symbols: corresponding absolute frequency of the inlet profile, shown over the absolutely unstable range of $S$. 
general trend is observed for jets with thinner initial shear layers, but as $R / \theta$ increases, the interval of $S$ over which the frequency selection criterion $\mathrm{St}_{g}=\mathrm{St}_{0}(x=0)$ may be regarded as valid is confined to an ever smaller vicinity of the global instability threshold. Note that the occurrence of vortex pairing, which distinguishes the configurations with $R / \theta \geqslant 15$ from those with $R / \theta=10$, is not associated with any sudden change in the global flow dynamics, in particular with respect to the frequency selection mechanism. The global frequencies close to onset in jets with $R / \theta=15$ are also remarkably well predicted by the absolute frequency at the inlet. At the highest value of $S$ for which synchronization is observed in an $R / \theta=20$ jet, the selected frequency still falls within 3\% of the expected value, but the frequency prediction degrades rapidly as $S$ is lowered. For $R / \theta=25$, only the $8 \%$ agreement between $\mathrm{St}_{0}$ and $\mathrm{St}_{g}$ at $S=0.6$ closest to threshold may still be considered satisfactory.

Tests have shown that the numerical values of $\mathrm{St}_{g}$ are quite sensitive to the choice of upstream boundary conditions. For instance, when the zero order characteristic boundary conditions (see Sec. III) were used in earlier simulations,${ }^{28}$ the global frequency of an $R / \theta=20, S=0.5$ jet was measured as $\mathrm{St}_{g}=0.443$, whereas the first order formulation employed in the present study leads to $\mathrm{St}_{g}=0.404$.

It should be noted that the only difference between the $R / \theta=10$ cases presented in Fig. 6 and those studied in Sec. V of Ref. 21 lies in the choice of the Reynolds number $(\mathrm{Re}=3750$ here, $\operatorname{Re}=1000$ in Ref. 21). The results are fully consistent: in the present study, global instability sets in at a slightly higher value of $S$, because the streamwise development of the base flow scales with Re, and the absolutely unstable region at a given temperature ratio is therefore 3.75 times as long as in the $\operatorname{Re}=1000$ case.

\section{B. Limited parameter range in the DNS}

In simulations of strongly heated thin shear layer jets, the pairing of large-scale vortices gives rise to an irregular ejection of free vortical structures into the outer flow. These structures visually resemble pictures of two-dimensional turbulence, and their generation is not a matter of numerical discretization, but seems to be a genuine feature of the axisymmetric equations of motion. Unsteady flow visualizations suggest that the formation of these structures results from a secondary instability due to the finely spun layer structure of rolled-up vortices in strongly stratified shear layers, which is documented in Sec. V below.

The pairing of two such layered vortices generates violent accelerations that may lead to folding and ejection of the outer vorticity layers. These folded layers then tend to form vorticity dipoles that are propelled into the outer flow through their self-induced motion. The dipoles are only slowly dissipated by viscosity and therefore may accumulate in the vicinity of the vortex pairing location. Such structures certainly would be highly unstable in a three-dimensional geometry, and their long-time dynamics observed in the axisymmetric simulations therefore should not be regarded as physical.

If the pairing takes place several jet diameters down- stream of the inlet, the eventual ejection of free vorticity dipoles into the outer flow has no impact on the global flow dynamics. In cases of strong heating and thin initial shear layers, however, the location of vortex pairing moves further upstream. The free vorticity dipoles may then contaminate the upstream boundary region and induce high-amplitude perturbations that disrupt the global flow synchronization. This behavior has been observed in three base flow configurations $(R / \theta=20, S=0.3),(R / \theta=25, S=0.3)$, and $(R / \theta=25$, $S=0.35$ ), and these cases therefore have been excluded from the presentation of results in Sec. IV A. Throughout the present study, only cases have been considered where the upstream region down to at least $x=4$ is free of spurious vorticity structures in the outer flow. The accessible ranges of the inlet velocity profile parameter $R / \theta$ and temperature ratio $S$ in the numerical simulation are limited due to this restriction.

\section{Comparison with experiments}

In Fig. 6, experimental values given by Monkewitz et $a l .{ }^{1}$ for the global frequency are included as grey symbols together with present numerical results. Only the "mode II" (Ref. 1) oscillating state is considered. According to a relation given in Ref. 1 , at a Reynolds number $\mathrm{Re}=3750$ based on the jet radius, the inlet conditions for these measurements should correspond to $R / \theta \sim 35$. The dimensional frequencies reported by Monkewitz et al. ${ }^{1}$ are scaled in Fig. 6 with respect to jet exit velocities that had to be deduced from the Reynolds number. The Mach number in these experiments varies within the interval $0.025<\mathrm{Ma}<0.05$.

Numerical simulations at $R / \theta=35$ were too contaminated with free vortices in the outer flow to be presented here. However, the experimentally measured Strouhal numbers align very well with the extrapolated trend of the numerical values as $R / \theta$ increases. A comparison with the linear instability properties of the base flow displays the same behavior that has been found in the simulations. Global instability almost coincides with the onset of absolute instability at the nozzle. At threshold, the global frequency $\mathrm{St}_{g}$ sets in $9 \%$ above the absolute frequency $\mathrm{St}_{0}$ (grey dashed line in Fig. 6), but while $\mathrm{St}_{0}$ decreases with stronger heating, $\mathrm{St}_{g}$ remains nearly constant over the covered range of temperature ratio $S$.

\section{Influence of acoustic feedback}

In several convectively unstable flow configurations, such as flow over cavities ${ }^{31}$ or jets impinging on an obstacle ${ }^{32}$ self-sustained oscillations are known to arise from the synchronization of a feedback loop consisting of a downstream-travelling vortical branch and an upstreamtravelling acoustic branch. In numerical simulations of compressible free jets, such a feedback loop may involve acoustic waves that are emitted from nonlinear vortex roll-up and pairing events, ${ }^{33,34}$ but also spurious acoustic noise generated at the numerical outflow has been reported to potentially give rise to unphysical self-excitation in numerical simulations of mixing layers. ${ }^{35}$ Grinstein et al. ${ }^{36}$ observed self-sustained os- 


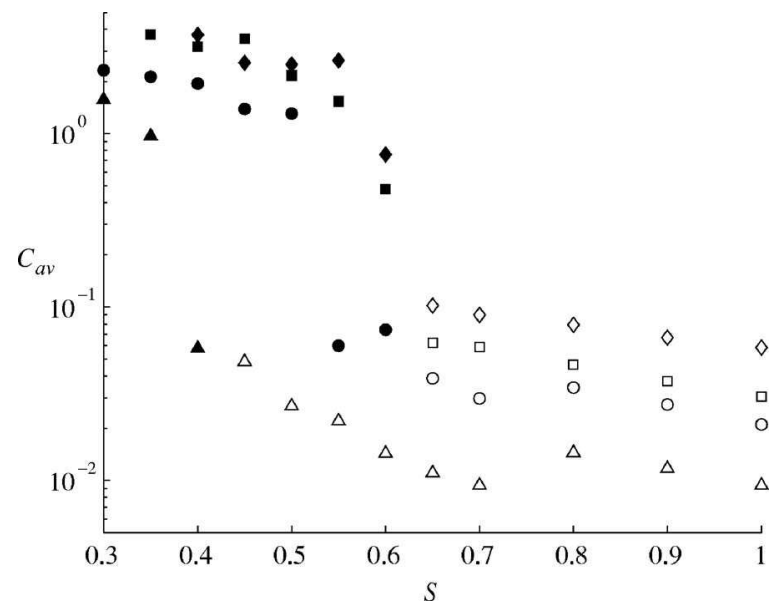

FIG. 7. Values of the conversion ratio $C_{\mathrm{av}}$, as defined in (3), as a function of $S$ for different values of $R / \theta$. Symbols as in Fig. 6: $\boldsymbol{\Delta}, \triangle R / \theta=10 ; \boldsymbol{\bullet}, \bigcirc$ $R / \theta=15 ; \square, \square R / \theta=20 ; \diamond, \diamond R / \theta=25$. Solid symbols denote synchronized cases, open symbols denote unsynchronized cases.

cillations due to acoustic feedback from vortex pairing in their simulation of a convectively unstable jet.

In the present simulations, in the absence of a hard nozzle, such acoustic feedback may only arise from spurious coupling between outgoing acoustic and incoming vortical waves at the numerical upstream boundary. Certainly the irregular perturbations that are observed to persist in simulations of entirely convectively unstable base flows must be the result of such spurious acoustic forcing, and these configurations therefore can be used to characterize the quality of the numerical upstream boundary conditions. Measures of typical conversion rates from outgoing acoustic to incoming vortical waves in these globally stable cases may then serve as a criterion to examine whether or not the synchronized oscillations observed in the globally unstable regime, considering their amplitude levels near the inlet, are likely to be influenced by spurious acoustic forcing.

Pressure fluctuations $p^{\prime}$ outside the jet at the upstream boundary $x=0$ are purely acoustic in nature, whereas $v^{\prime}$ fluctuations inside the shear layer are strongly dominated by vortical instability waves. An ad hoc measure of the acoustic-vorticity conversion at the inlet in a convectively unstable flow may be defined as the ratio

$$
C_{\mathrm{av}}=\frac{v_{\mathrm{rms}}^{\prime}(r=1, x=1)}{p_{\mathrm{rms}}^{\prime}(r=2, x=0)},
$$

evaluated in the asymptotic regime. Values of $C_{\mathrm{av}}$ for all flow cases are given in Fig. 7. In all globally stable configurations (open symbols in Fig. 7, compare to Fig. 4), $C_{\text {av }}$ varies between $10^{-2}$ and $10^{-1}$. This order of magnitude seems to be characteristic of situations where instabilities are driven by acoustic forcing at the inlet. Such forcing naturally is more efficient in thin shear layer jets, but for constant values of $R / \theta$ it is found to vary only slowly with $S$ in the globally stable regime. In contrast, $C_{\mathrm{av}}$ takes on significantly higher values in most configurations exhibiting synchronized oscillations. The $v^{\prime}$ fluctuations in these cases are too strong to be the result of acoustic forcing, and the noise-driven oscilla- tions therefore appear to have been replaced by a global mode induced by absolute instability.

According to Fig. 7, one might wonder whether the cases $(R / \theta=10, S=0.4)$ and $(R / \theta=15, S=0.55)$ are likely to be dominated by acoustic feedback. We feel that these two marginal cases quite accurately mark the onset of a supercritical bifurcation. The configuration $(R / \theta=15, S=0.6)$ is atypical: although it marks the onset of self-sustained oscillations, this threshold does not coincide with a significant increase of $C_{\mathrm{av}}$. This observation suggests that acoustic feedback is indeed involved in the self-excited behavior. However, the inlet profile in this case is already absolutely unstable, and the global frequency has been found to very accurately obey the selection criterion for a global mode (see Fig. 6). We therefore believe this flow configuration to exhibit the behavior of a slightly damped oscillator, in the sense of Huerre and Monkewitz; ${ }^{2}$ in the absence of any upstream forcing, such a flow would be globally stable, but very close to threshold. The low-level acoustic forcing introduced by the numerical boundary conditions seems to be sufficient to destabilize the otherwise slightly damped global mode. Note that the "mode 2" oscillatory states reported in Ref. 21 bear the same characteristic features as the one observed here in the $(R / \theta=15, S=0.6)$ base flow. It may be surmised that the occurrence of these "mode 2 " states ${ }^{21}$ are also the manifestation of a slightly damped global mode destabilized by low-level acoustic feedback.

\section{INNER STRUCTURE OF ROLLED-UP STRATIFIED VORTICES}

In an isothermal jet, vortex roll-up is a process involving only the redistribution and viscous dissipation of the vorticity initially contained in the unperturbed shear layer. Figure 8 (a) shows a vortex rolling up in a perturbed isothermal jet with $R / \theta=10$. The action of viscosity leads to a smooth vorticity distribution inside the vortex core, and to a preferred dissipation in the thin braids that connect neighboring vortices. No negative vorticity is produced. In contrast, in the regime of low $S$, where global instability is observed, the simulations display a more complex inner structure of the rolled-up vortex billows, as demonstrated in Fig. 8(b) for a typical newly formed vortex in a jet with $R / \theta=10$ and $S$ $=0.4$. Isocontours of the total vorticity $\Omega=\Omega_{b}+\Omega^{\prime}$ are shown. The rolled-up sheets of positive vorticity from the initial shear layer are interlaced with regions of negative vorticity, and their absorption into the compact vortex core takes place on a slower time scale than in the isothermal jet.

In the context of nonisothermal planar shear layers, Klaassen and Peltier ${ }^{37,38}$ conclude that baroclinic production of vorticity in the vortex braids may dominate over viscous dissipation if the temperature gradient is sufficiently strong. Thin layers of high vorticity from the braids are then successively wrapped around the vortex core, forming a more pronounced spiral sheet structure than is found in isothermal shear layers. In Refs. 37 and 38, the braid regions are identified as the prevalent site of baroclinic vorticity production.

A closer investigation of the vortex roll-up process in the heated jet confirms that the baroclinic torque is responsible 
(a)

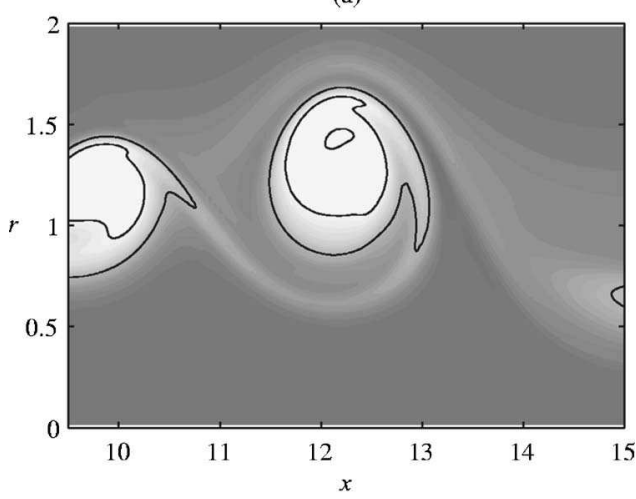

(b)

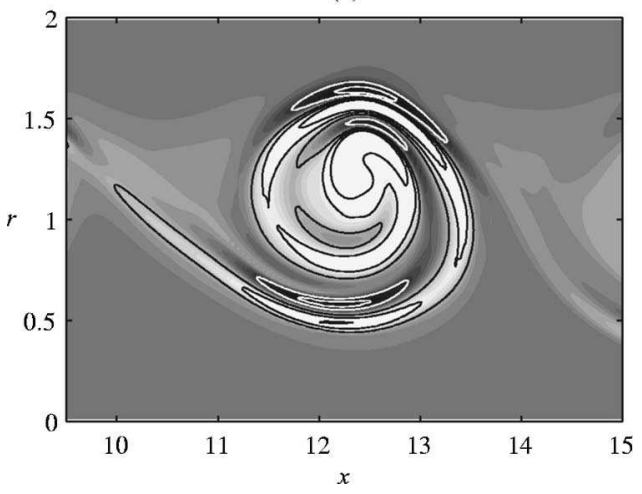

FIG. 8. Vortex roll-up in jets with parameters (a) $R / \theta=10, S=1$ (isothermal); (b) $R / \theta=10, S=0.5$ (heated). Vorticity isocontours: Light shading and black lines are positive, dark shading and white lines are negative.

for the formation of the layered vorticity structure. Figure 9(a) displays the total density distribution corresponding to Fig. 8(b). The vortex billow is composed of two entwined spiraling fingers of high and low density fluid. Mixing between these two densities only occurs in the very core of the vortex. The vorticity layers displayed in Fig. 8(b) are located on the interfaces between regions of high and low density. The density and pressure gradients are misaligned, and therefore exert a baroclinic torque $(\nabla \rho \times \nabla p) / \rho^{2}$, mapped in Fig. 9(b). A fluid particle on an anticlockwise trajectory around the center of the vortex successively passes through alternating regions of positive and negative baroclinic torque. Such alternating regions in Fig. 9(b) are perfectly aligned with the outer vorticity layers displayed in Fig. 9(a). Written in total flow quantities, the vorticity equation reads

$$
D_{t} \Omega=-\Omega\left(\partial_{x} u+\partial_{r} v\right)+\frac{\nabla \rho \times \nabla p}{\rho^{2}}+\operatorname{curl}\left(\frac{\operatorname{div} \tau}{\rho}\right) .
$$

The distribution of the viscous dissipation term is displayed in Fig. 9(c). Dissipation is concentrated in layers that align with those of Fig. 8(b). The material derivative $D_{t} \Omega=\partial_{t} \Omega$ $+\nabla \Omega \cdot \mathbf{u}$ is presented in Fig. 9(d). Comparison with Fig. $9(\mathrm{~b})$ clearly demonstrates that the dominant contribution to $D_{t} \Omega$ in the outer layers of the vortex is provided by the baroclinic torque. However, in contrast to the study of Klaassen and Peltier, ${ }^{37,38}$ the vorticity production does not take place in the braids between neighboring vortices, but inside the vortex billow. (a)

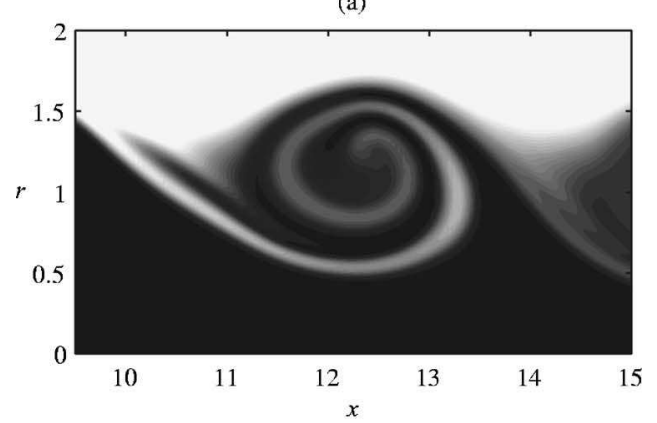

(b)

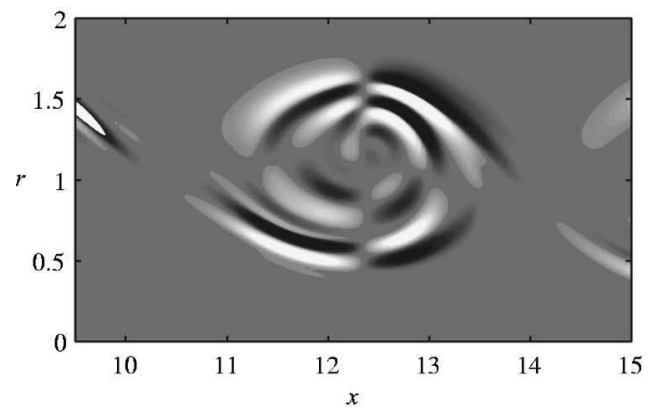

(c)

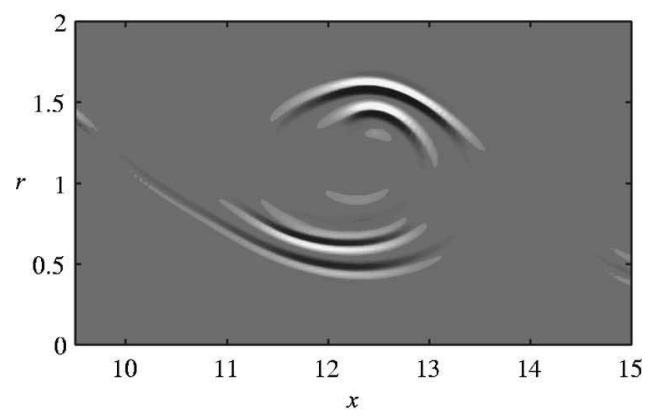

(d)

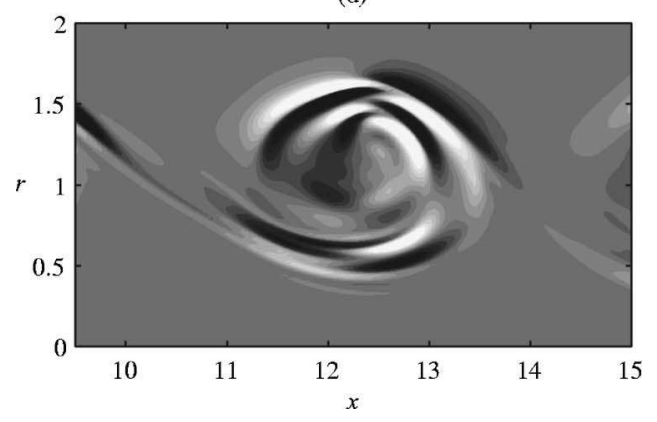

FIG. 9. (a) Total density: black is light fluid, white is heavy fluid. (b) Baroclinic torque; (c) viscous vorticity dissipation; (d) total vorticity production $D_{t} \Omega$. Grey scale values are identical in (b)-(d): light is positive, dark is negative. All snapshots are taken at the same time as in Fig. 8(b).

\section{CONCLUSION}

The global stability of hot round jets has been examined via direct numerical simulation of the axisymmetric equations of motion. The shear layer thickness of the inlet profile and the ambient-to-jet temperature ratio have been systematically varied over the ranges $10 \leqslant R / \theta \leqslant 25$ and $0.3 \leqslant S$ $\leqslant 1$. Globally unstable situations are characterized by the onset of self-sustained synchronized oscillations that give rise to highly regular ring vortices. 
The numerical results have clearly demonstrated that global instability, over the investigated parameter range, is associated with the presence of a finite region of local absolute instability. No synchronized oscillations have been found to persist in flow configurations that are convectively unstable everywhere. At any fixed value of $R / \theta$, global instability has been observed to set in at a temperature ratio just slightly below the critical value at which local absolute instability starts to develop at the upstream boundary of the flow domain. As $S$ is lowered further beyond the global instability threshold, and the streamwise extent of the absolutely unstable flow region consequently grows larger, the flow never returns to a globally stable behavior. These numerical observations are in excellent agreement with the theoretical model of nonlinear global modes in a semi-infinite domain described by Couairon and Chomaz. ${ }^{5}$ Spurious excitation from upstream-travelling acoustic waves does not qualify as the driving mechanism of the flow synchronization. Its influence seems to be limited to a possible slight destabilization of flows on the brink of global instability.

It has further been shown that the frequencies of globally unstable jets obey the theoretical predictions: ${ }^{5}$ at threshold, the global frequency of the thickest shear layer jet $(R / \theta$ $=10$ ) matches the absolute frequency at the inlet within $0.5 \%$ accuracy. An agreement of $8 \%$ is found in the thinnest shear layer jet considered $(R / \theta=25)$. The conclusions drawn in Ref. 21 from the investigation of jets with $R / \theta=10$ and over a range of temperature ratios $0.1 \leqslant S \leqslant 1$ are found to hold true also in jets with thinner initial shear layers. However, the numerical results indicate that in thin shear layer jets the validity of the theoretical frequency selection criterion ${ }^{5}$ is restricted to the immediate vicinity of the global instability threshold.

The numerical observations pertaining to the onset of global instability and the selection of the global frequency seem to be consistent with the "mode II" oscillations reported in the experiments of Monkewitz et al. ${ }^{1}$ In agreement with Kyle and Sreenivasan, ${ }^{12}$ no oscillating states have been detected that would correspond to "mode I" of Ref. 1. The comparison remains qualitative, because the experimentally investigated range $R / \theta \geqslant 35$ (Refs. 1 and 12) could not be attained in the present study. Above the upper limit $R / \theta$ $=25$, free vortical structures in the outer flow quickly contaminate the numerical inflow region.

The inner structure of the rolled-up ring vortices in hot jets has been documented. It is characterized by thin vorticity layers of alternating sign, wrapped around a compact core. This layer structure has been shown to arise from vorticity production due to the baroclinic torque.

It may be concluded that the nonlinear global mode model of Ref. 5 accurately describes the physical mechanism that causes the onset of global instability in hot jets: a nonlinear wave front moves upstream in an absolutely unstable environment, until it is blocked at the upstream boundary. In the asymptotic state, the front then imparts its linearly selected frequency to the entire flow. In supercritical flow situations, the model ${ }^{5}$ foresees a departure of the global frequency from the absolute frequency, as the spatial envelope of the oscillations must satisfy a matching condition between a region of exponential growth and the upstream boundary conditions. Whether this mechanism alone accounts for the numerically observed discrepancy between absolute and global frequencies in highly supercritical thin shear layer jets remains to be clarified.

\section{ACKNOWLEDGMENTS}

The authors gratefully acknowledge the advice and support of Marc Terracol in the development of the numerical code. Many valuable discussions with Jean-Marc Chomaz have contributed to this study. Computational resources were provided by ONERA. Lutz Lesshafft has been supported by a Ph.D. fellowship from the EADS Foundation.

${ }^{1}$ P. A. Monkewitz, D. W. Bechert, B. Barsikow, and B. Lehmann, "Selfexcited oscillations and mixing in a heated round jet," J. Fluid Mech. 213, 611 (1990).

${ }^{2}$ P. Huerre and P. A. Monkewitz, "Local and global instabilities in spatially developing flows," Annu. Rev. Fluid Mech. 22, 473 (1990).

${ }^{3}$ J.-M. Chomaz, "Global instabilities in spatially developing flows: Nonnormality and nonlinearity," Annu. Rev. Fluid Mech. 37, 357 (2005).

${ }^{4}$ M. Provansal, C. Mathis, and L. Boyer, "Bénard-von Kármán instability: transient and forced regimes," J. Fluid Mech. 182, 1 (1987).

${ }^{5}$ A. Couairon and J.-M. Chomaz, "Fully nonlinear global modes in slowly varying flows," Phys. Fluids 11, 3688 (1999).

${ }^{6}$ B. Pier and P. Huerre, "Nonlinear self-sustained structures and fronts in spatially developing wake flows," J. Fluid Mech. 435, 145 (2001).

${ }^{7} \mathrm{~B}$. Pier, "On the frequency selection of finite-amplitude vortex shedding in the cylinder wake," J. Fluid Mech. 458, 407 (2002).

${ }^{8}$ P. Huerre and P. A. Monkewitz, "Absolute and convective instabilities in free shear layers,” J. Fluid Mech. 159, 151 (1985).

${ }^{9}$ F. Gallaire, M. Ruith, E. Meiburg, J.-M. Chomaz, and P. Huerre, "Spiral vortex breakdown as a global mode," J. Fluid Mech. 549, 71 (2006).

${ }^{10}$ P. J. Strykowski and D. L. Niccum, "The stability of countercurrent mixing layers in circular jets," J. Fluid Mech. 227, 309 (1991).

${ }^{11}$ S. Jendoubi and P. J. Strykowski, "Absolute and convective instability of axisymmetric jets with external flow," Phys. Fluids 6, 3000 (1994).

${ }^{12}$ D. Kyle and K. Sreenivasan, "The instability and breakdown of a round variable-density jet," J. Fluid Mech. 249, 619 (1993).

${ }^{13}$ K. Sreenivasan, S. Raghu, and D. Kyle, "Absolute instability in variable density round jets,” Exp. Fluids 7, 309 (1989).

${ }^{14}$ C. Richards, B. Breuel, R. Clark, and T. Troutt, "Concentration measurements in a self-excited jet," Exp. Fluids 21, 103 (1996).

${ }^{15}$ B. Yildirim and A. Agrawal, "Full-field measurements of self-excited oscillations in momentum-dominated helium jets," Exp. Fluids 38, 161 (2005).

${ }^{16}$ P. A. Monkewitz and K. Sohn, "Absolute instability in hot jets," AIAA J. 26, 911 (1988).

${ }^{17}$ L. Lesshafft and P. Huerre, "Linear impulse response in hot round jets," Phys. Fluids 19, 024102 (2007).

${ }^{18}$ A. Couairon and J.-M. Chomaz, "Global instability in fully nonlinear systems," Phys. Rev. Lett. 77, 4015 (1996).

${ }^{19}$ A. Couairon and J.-M. Chomaz, "Absolute and convective instabilities, front velocities and global modes in nonlinear systems," Physica D 108, 236 (1997).

${ }^{20}$ J.-M. Chomaz, "Fully nonlinear dynamics of parallel wakes," J. Fluid Mech. 495, 57 (2003).

${ }^{21}$ L. Lesshafft, P. Huerre, P. Sagaut, and M. Terracol, "Nonlinear global modes in hot jets," J. Fluid Mech. 554, 393 (2006).

${ }^{22}$ A. Michalke, "Instabilität eines kompressiblen runden Freistrahls unter Berücksichtigung des Einflusses der Strahlgrenzschichtdicke," Z. Flugwiss. 19, 319 (1971), English translation: NASA Technical Memo 75190 (1977)

${ }^{23} \mathrm{G}$. Lu and S. Lele, "A numerical investigation of skewed mixing layers," Technical Report No. TF-67, Department of Mechanical Engineering, Stanford University, Stanford, CA (1996).

${ }^{24}$ S. Lele, "Compact finite difference schemes with spectral-like resolution," J. Comput. Phys. 103, 16 (1992).

${ }^{25} \mathrm{M}$. Visbal and D. Gaitonde, "On the use of higher-order finite-difference schemes on curvilinear and deforming meshes," J. Comput. Phys. 181, 
155 (2002).

${ }^{26}$ M. B. Giles, "Nonreflecting boundary conditions for Euler equation calculations," AIAA J. 28, 2050 (1990).

${ }^{27}$ T. Colonius, S. K. Lele, and P. Moin, "Boundary conditions for direct computation of aerodynamic sound generation," AIAA J. 31, 1574 (1993).

${ }^{28}$ L. Lesshafft, P. Huerre, P. Sagaut, and M. Terracol, "Global modes in hot jets, absolute/convective instabilities and acoustic feedback," AIAA Pap. 2005-3040 (2005).

${ }^{29}$ C. Bogey, C. Bailly, and D. Juvé, "Noise investigation of a high subsonic, moderate Reynolds number jet using a compressible large eddy simulation," Theor. Comput. Fluid Dyn. 16, 273 (2003).

${ }^{30} \mathrm{P}$. A. Monkewitz, "On the nature of the amplitude modulation of jet shear layer instability waves," Phys. Fluids 26, 3180 (1983).

${ }^{31}$ L. Larchevêque, P. Sagaut, T.-H. Lê, and P. Comte, "Large-eddy simulation of a compressible flow in a three-dimensional open cavity at high Reynolds number," J. Fluid Mech. 516, 265 (2004).
${ }^{32}$ C.-M. Ho and N. S. Nosseir, "Dynamics of an impinging jet. Part 1. The feedback phenomenon,” J. Fluid Mech. 105, 119 (1981).

${ }^{33}$ J. Laufer and P. A. Monkewitz, "On turbulent jet flows: A new perspective," AIAA Pap. 80-0962 (1980).

${ }^{34}$ C.-M. Ho and P. Huerre, "Perturbed free shear layers," Annu. Rev. Fluid Mech. 16, 365 (1984).

${ }^{35} \mathrm{~T}$. Colonius, "Modeling artificial boundary conditions for compressible flow," Annu. Rev. Fluid Mech. 36, 315 (2004).

${ }^{36}$ F. F. Grinstein, E. S. Oran, and J. P. Boris, "Direct numerical simulation of axisymmetric jets," AIAA J. 25, 92 (1987).

${ }^{37}$ G. P. Klaassen and W. R. Peltier, "Evolution of finite amplitude KelvinHelmholtz billows in two spatial dimensions," J. Atmos. Sci. 42, 1321 (1985).

${ }^{38}$ G. P. Klaassen and W. R. Peltier, "The influence of stratification on secondary instability in free shear layers," J. Fluid Mech. 227, 71 (1991). 